\title{
A Novel Design and Implementation of new Peres and Six- Correction (PSCL) gate in Quantum-dot Cellular Automata (QCA) Technology
}

\author{
Md. Jahangir Alam ${ }^{1}$, Ranajit Mandal ${ }^{2}$ \\ ${ }^{1}$ Department of Computer Science and Engineering, \\ Mawlana Bhashani Science and Technology University, \\ Department of ICT \\ Bangladesh \\ ${ }^{2}$ Department of Computer Science and Engineering, \\ Mawlana Bhashani Science and Technology University, \\ Department of ICT \\ Bangladesh
}

\begin{abstract}
In recent years Quantum cellular automata (QCA) have been used widely to digital circuits and systems. QCA technology is a promising alternative to CMOS technology. It is attractive due to its fast speed, small area and low power consumption. The QCA offers a novel electronics paradigm for information processing and communication. It has the potential for attractive features such as faster speed, higher scale integration, higher switching frequency, smaller size and low power consumption than transistor based technology. In this paper, new Peres and Six-Correction (PSCL) gate is proposed based on QCA logic gates: the PSCL gate, MV gate and Inverter gate. The proposed circuit is a promising future in constructing of nano-scale low power consumption information processing system and can stimulate higher digital applications in QCA.
\end{abstract}

Keywords: Quantum Cellular Automata; QCA Logic Gates; PSCL gate.

\section{Introduction}

Quantum technology has gradually applied in various fields [1,2]. Quantum-dot cellular automata is projected as a promising nanotechnology for future ICs [3,4]. A QCA is an array of structures known as quantum-dots. Computing with QCA is achieved by the tunneling of individual electrons among the quantum-dots inside a cell and the classical coulomb interaction among them. A QCA cell consists of two electrons positioned at opposite corners owing to columbic repulsion, so the polarization states of $\mathrm{P}=-1$ and $\mathrm{P}=+1$ can be represented by two stable configuration of a pair of electrons, the corresponding the logic values of " 0 " and " 1 " also be represented in Fig. 1 The electrostatic repulsion between electrons leads to the synchronization of neighboring cells. Thus, one cell's polarization is determined by the effect of its neighboring cell's polarization.

Therefore, the array of QCA cells will be able to propagate information as a wire [5]. The QCA cells can form the primitive logic gates shown in Fig. 2 (inverter gate), Fig. 3 (majority gate). A majority gate with the logic function of $\mathrm{MV}(\mathrm{A}, \mathrm{B}, \mathrm{C})=\mathrm{AB}+\mathrm{AC}+\mathrm{BC}$ is composed of five cells. By setting one of the inputs of this gate permanently to 0 or 1 , AND and OR functions will be formed in QCA. Some other combinational logic designs with plus-shaped quantum-dot cellular automata using minority gate as the fundamental building block have been presented in [6].

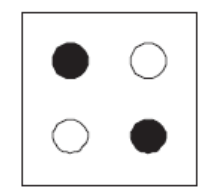

Binary "0"

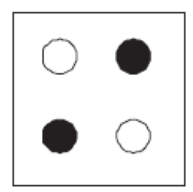

Binary "1"
Fig. 1Quantum cellular automata

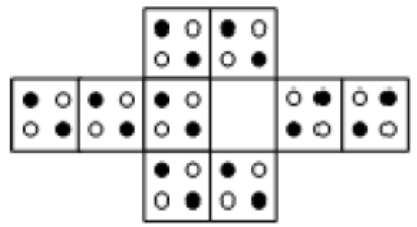

Fig. 2 Inverter gate 
Input $A$

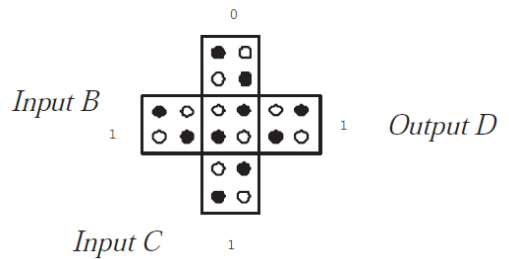

Fig. 3 Majority gate

An array of cells that are aligned can construct a QCA wire which is shown in Fig.4. The polarization of each cell in a QCA wire is directly affected by the polarization of its neighboring cells on account of electrostatic force. Accordingly, QCA wires can be used to propagate information from one end to another [7](Kim et al., 2007).(SCL Paper Reference)

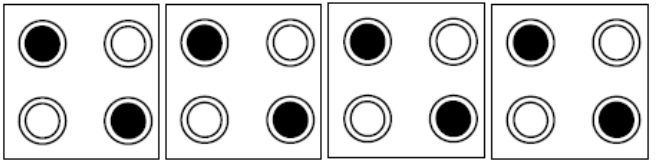

Fig. 4 A QCA Wire

\section{Six-Correction Logic Gate}

Six-Correction Logic Gate is a 4 x 4 gate with two garbage outputs [8] (Bhagyalakshmi, et al., 2010), the input vector is $\mathrm{I}(\mathrm{A}, \mathrm{B}, \mathrm{C}, \mathrm{D})$ and the output vector is $\mathrm{O}(\mathrm{P}$, $\mathrm{Q}, \mathrm{R}, \mathrm{S}$ ) and output is defined by $\mathrm{P}=\mathrm{A}, \mathrm{Q}=\mathrm{B}, \mathrm{R}=\mathrm{C}$, $\mathrm{S}=\mathrm{A}(\mathrm{B}+\mathrm{C}) \oplus \mathrm{D}$ the relation between input and output shown in Fig.5. There is a one-to-one mapping between inputs and outputs of SCL gate and it can be used to add 6 to the sum in order to correct it to get the correct BCD sum [8] (Bhagyalakshmi, et al., 2010).

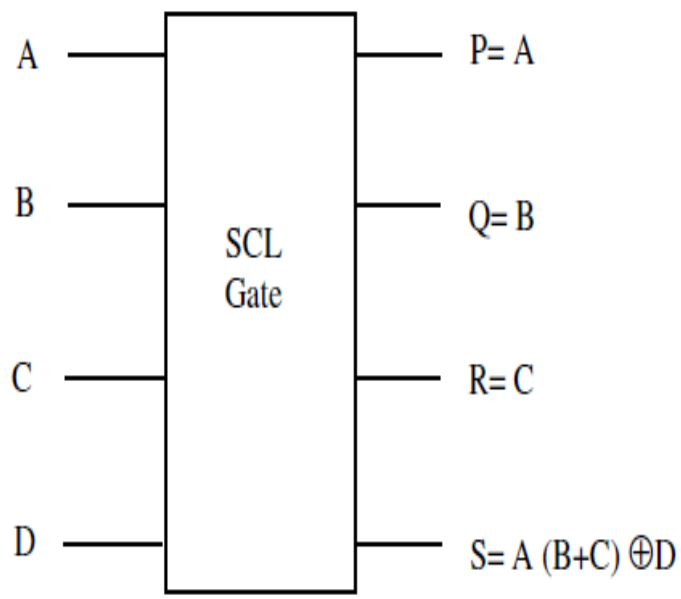

Fig.5 Six-correction logic gate (SCLG)

\subsection{Double Feynman gate (F2G)}

Fig.6 shows a $3 \times 3$ Peres gate [9]. The input vector is $I(A$, $\mathrm{B}, \mathrm{C})$ and the output vector is $\mathrm{O}(\mathrm{P}, \mathrm{Q}$ and $\mathrm{R})$. The output is defined by $\mathrm{P}=\mathrm{A}, \mathrm{Q}=\mathrm{A} \oplus \mathrm{B}$ and $\mathrm{R}=\mathrm{AB} \oplus \mathrm{C}$.

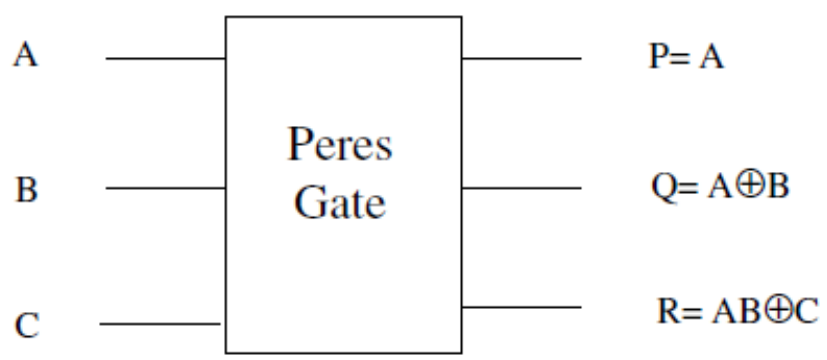

Fig.6 Peres gate

\section{Proposed Peres and Six-Correction (PSCL) Gate}

\section{PSCL gate}

Fig.7 shows a PSCL Gate. The input vector is I (A, B, C, $\mathrm{D})$ and the output vector is $\mathrm{O}(\mathrm{P}, \mathrm{Q}, \mathrm{R}, \mathrm{S}) . \mathrm{P}=\mathrm{A}, \mathrm{Q}=\mathrm{A} \oplus \mathrm{B}$, $\mathrm{R}=\mathrm{C}$ and $\mathrm{S}=\mathrm{A}(\mathrm{B}+\mathrm{C}) \oplus \mathrm{D}$.
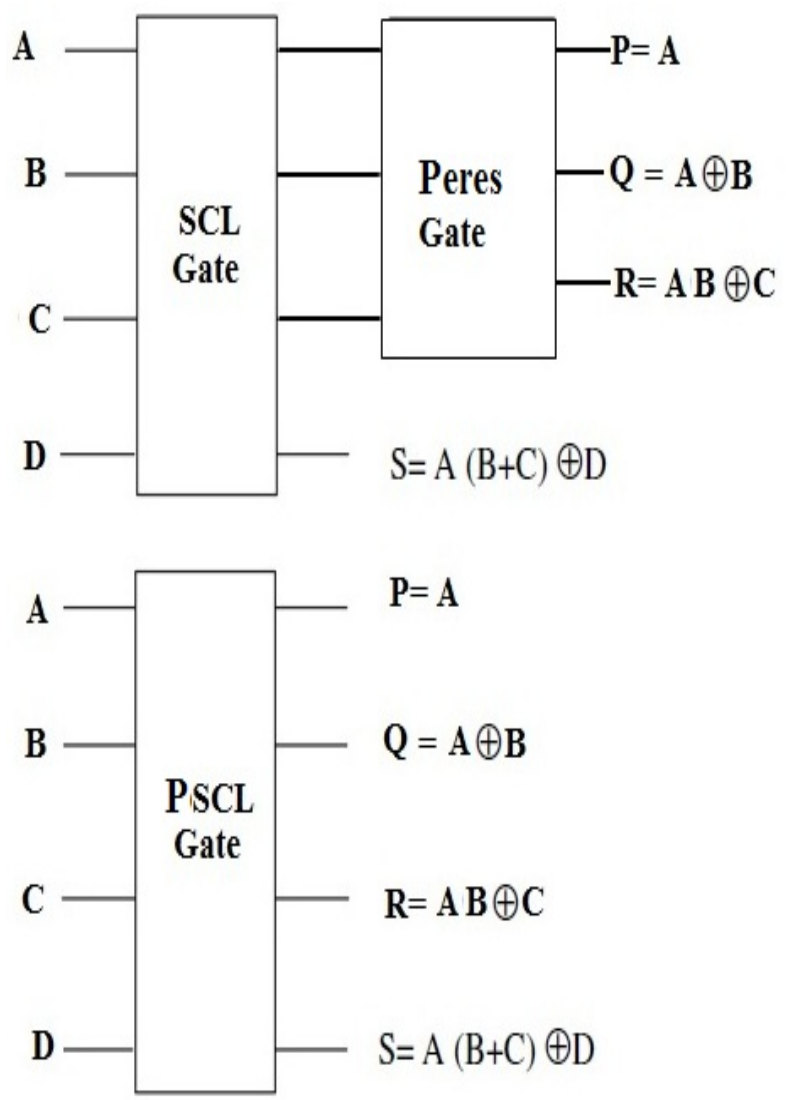

Fig.7 PSCL Gate 
Fig.8 shows the QCA representation of Peres and SixCorrection Logic Gate (PSCL) based of majority voter (MV) gate. Here twelve majority voter gates are used to design Six-correction logic gate (PSCL).

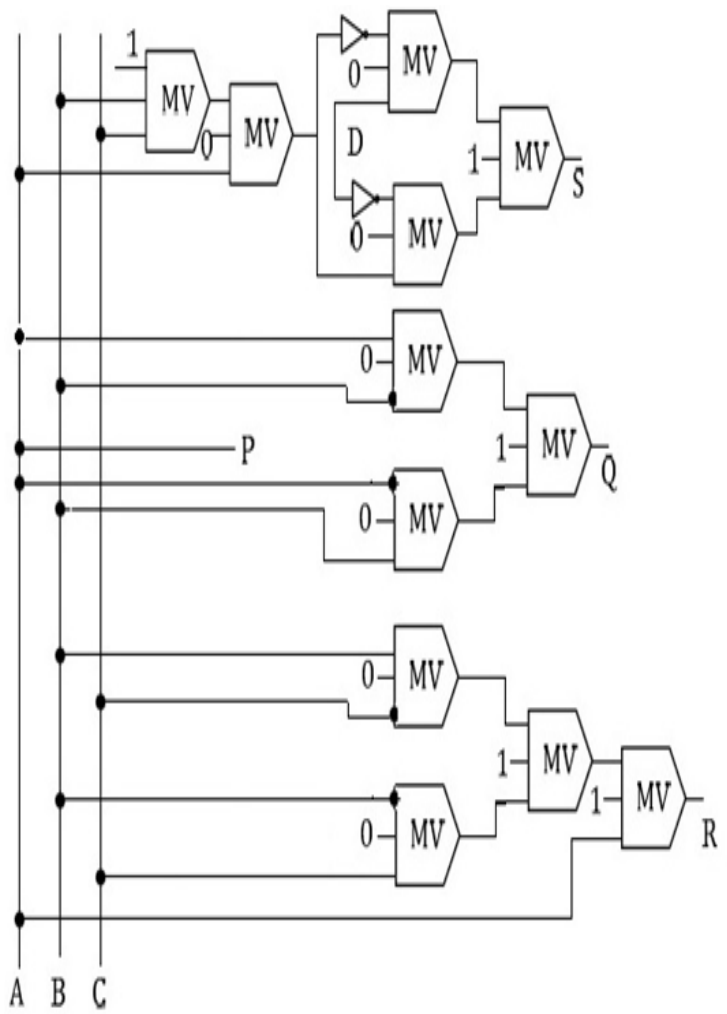

Fig.8 QCA block diagram of PSCL gate

\section{Simulation and Result Discussion}

The circuit is functionally simulated using the QCADesigner [10] (Walus et al., 2004). The simulated circuit layout is shown in figure 6 , here the input signals are: $\mathrm{A}, \mathrm{B}, \mathrm{C}$ and $\mathrm{D}$ and the output signals are: $\mathrm{P}=\mathrm{A}, \mathrm{Q}=\mathrm{B}$, $\mathrm{R}=\mathrm{C}$ and $\mathrm{S}=\mathrm{A}(\mathrm{B}+\mathrm{C}) \oplus \mathrm{D}$ and this module goes through four clock zones, it means that the delay is a full clock cycle. Therefore, at the output $\mathrm{P}, \mathrm{Q}, \mathrm{R}$ and $\mathrm{S}$ are available one clock cycles after A, B, C and D has been applied. Moreover, it requires sixty one (61) cells and total area of $0.095 \mu \mathrm{m} 2$.

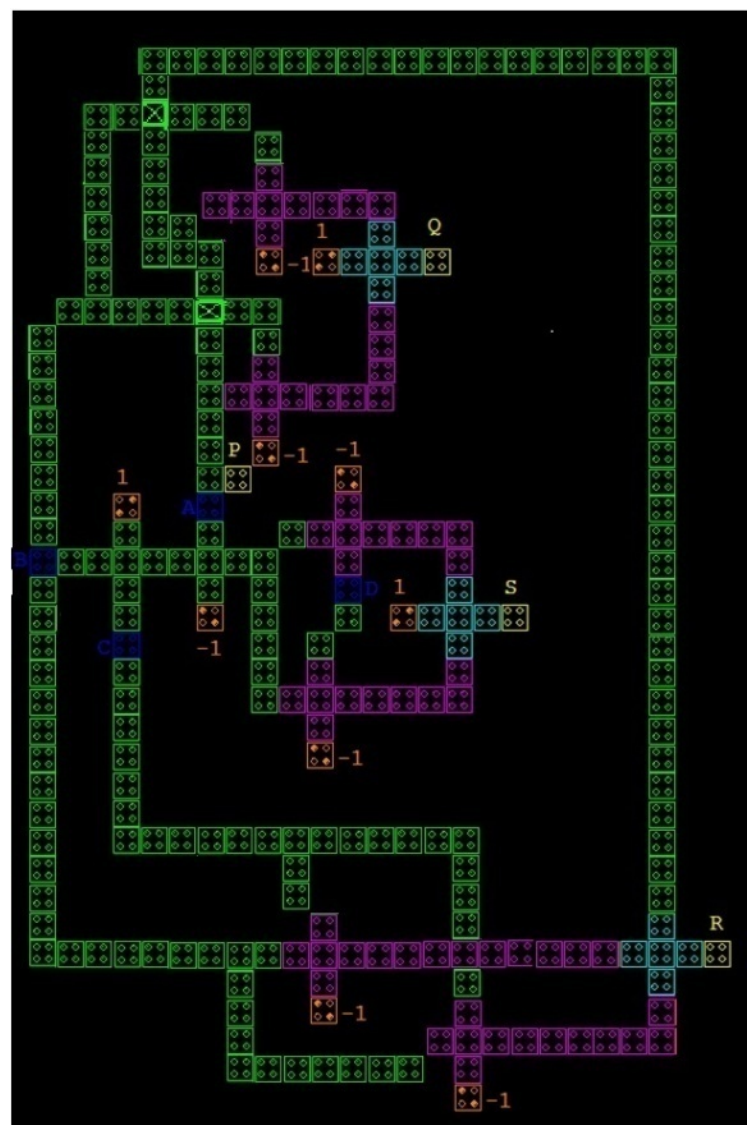

Fig.9 QCA circuit layout of PSCl logic gate

We can find the output value of $\mathrm{S}$ is low level when the input digits $(\mathrm{A}=0, \mathrm{~B}=0, \mathrm{C}=0, \mathrm{D}=0)$ and $\mathrm{S}$ is up level when the input digits $(\mathrm{A}=0, \mathrm{~B}=0, \mathrm{C}=0, \mathrm{D}=1)$. We look into the other two output values of $\mathrm{A}, \mathrm{B}$ and $\mathrm{C}$ also translating the input data successfully. The simulated waveforms of PSCL gate is shown in Fig.10. Here, the S output is delayed by 0.75 clock cycle. 


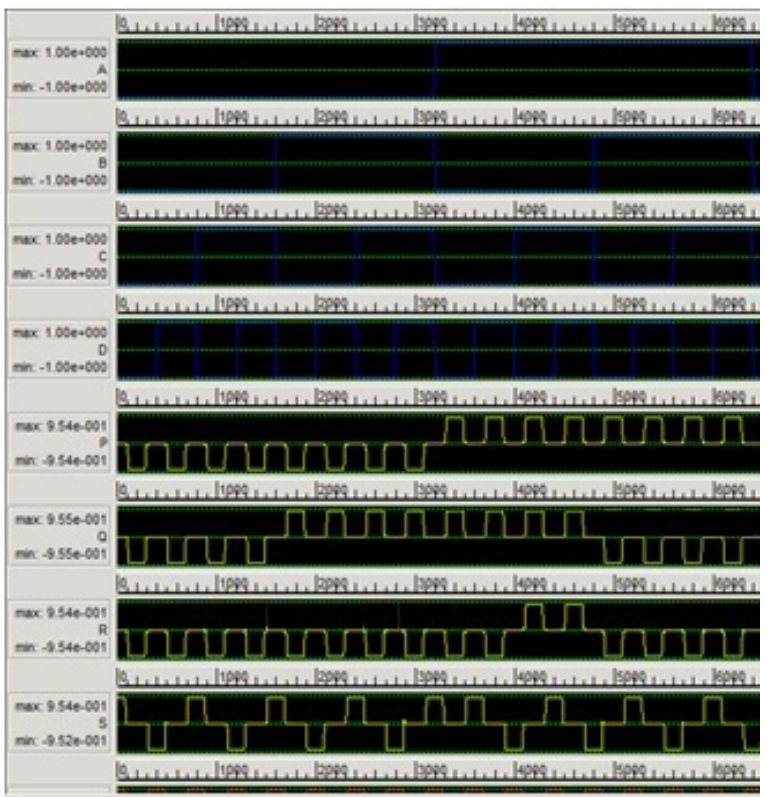

Fig.10 Simulated waveforms for PSCL gate circuit.

\section{CONCLUSIONS}

This paper present Peres and Six-correction logic (PSCL) gate based on QCA does logic gates. The proposed PSCL gate has been simulated and verified using QCADesigner. The result is compared in terms of complexity (cell count), covered area and time delay. The simulation result shows that the proposed design achieves a sound improvement. This design will be very helpful for designing ultra low power digital circuits.

\section{References}

[1] Yi Liu: Modified Quantum Genetic Algorithm Apply for Flow Shop Scheduling Problem. Journal of Computational Information Systems. Vol. 4 (2008), pp. 183 - 188.

[2] Hao Li, Shiyong Li: A Quantum Immune Evolutionary Algorithm and Its Application. Journal of Computational Information Systems. Vol. 7 (2011), pp. 2972 - 2979.

[3] Lent, C. S., Tougaw, P. D., and Prod, W.: Quantum Cellular Automata: The physics of computing with quantum dot molecules. Physics and Computation (1994), pp. 5 - 13.

[4] C. Lent, and P. Tougaw: A device architecture for computing with quantum dots. Proceedings of the IEEE. vol. 85 (1997), pp. $541-557$.

[5] Lent, Craig S., Tougaw, P. Douglas: Lines of interacting quantum-dot cells: A binary wire. Journal of Applied Physics. vol. 74 (1993), pp. $6227-6233$.
[6] Samir Roy, Biswajit Saha: Minority gate oriented logic design with quantum-dot cellular automata. Lecture Notes in Computer Science. vol. 4173 (2006), pp. 646 - 656.

[7] Kim, K., Wu, K., and Karri, R. 2007. The robust QCA adder designs using composable QCA building blocks. ComputerAided Design of Integrated Circuits and Systems, IEEE Transactions on 26(1): 176-183.

[8] Bhagyalakshmi, H. R., and Venkatesha, M. K. (2010). Optimized reversible BCD adder using new reversible logic gates. arXiv preprint arXiv:1002.3994.

[9] A. Peres, Reversible logic and quantum computers, Physical Review A 32 (6) (1985) 3266-3276.

[10] Walus K. QCA Designer. QCA Designer website. University of Calgary ATIPS Laboratory. http://www.qcadesigner.ca.

Md. Jahangir Alam is currently completing his M. Engg in the Department of Computer Science and Engineering, Mawlana Bhashani Science and Technology University, Santosh, Tangail 1902, Bangladesh. He has completed his B. Sc in the Department of Computer Science and Engineering at the same university. His area of research interest is cloud computing, reversible logic synthesis etc.

Ranajit Mandal is currently completing his M. Engg in the Department of Computer Science and Engineering, Mawlana Bhashani Science and Technology University, Santosh, Tangail 1902, Bangladesh. He has completed his B. Sc in the Department of Computer Science and Engineering at the same university. His area of research interest is cloud computing, reversible logic synthesis etc.. 